\title{
Überprüfung und Bewertung des Schriftbildes sowie des Anlagenzustandes industrieller Signieranlagen mit maschinellem Lernen
}

\author{
Nicole Immesberger ${ }^{1}$, Steffen Klein ${ }^{1}$, Martin Jochum ${ }^{2}$, Andreas Schütze ${ }^{1}$ \\ 1 ZeMA - Zentrum für Mechatronik und Automatisierungstechnik gGmbH \\ Eschberger Weg 46, 66121 Saarbrücken, Deutschland \\ 2 Aktien-Gesellschaft der Dillinger Hüttenwerke \\ Werkstraße 1, 66763 Dillingen/Saar, Deutschland
}

\begin{abstract}
Zusammenfassung
Ungünstige Prozesssituationen bedingt durch einen fehlerbehafteten Anlagenzustand zeigen sich bei Markieranlagen nicht nur in den Signalen interner Anlagensensorik, sondern auch in Anomalien des Schriftbildes der Markierung. In dieser Arbeit wird daher ein Ansatz zur Schriftbildkontrolle und Anlagenzustandsüberwachung von Blechmarkieranlagen vorgestellt. Auf Basis von Kameraaufnahmen wird das Signierergebnis automatisch gemäß Markiervorgabe auf Vollständigkeit und Qualität beurteilt. Die Aufnahmen durchlaufen dazu eine Signalverarbeitungskette, welche sich aus Schritten der digitalen Bildverarbeitung und Methoden des maschinellen Lernens zusammensetzt. Unerwünschte Effekte können dabei durch gezielte Merkmalsextraktion und -selektion von Bildattributen detektiert und eingeordnet werden. Aus der jeweiligen Klassenzugehörigkeit lassen sich prädiktive Maßnahmen für die Erhaltung eines einwandfreien Anlagenzustandes und optimalen Signierergebnisses ableiten.
\end{abstract}

Keywords: Bildverarbeitung, Zustandsüberwachung, Maschinelles Lernen, Schriftbildkontrolle, PunktMatrix-Schrift

\begin{abstract}
Motivation und Einleitung
Anlagenzustandsüberwachung mit dem Ziel der frühzeitigen Fehlererkennung in Prozessen und Ableitung von Maßnahmen zur bedarfsgerechten Instandhaltung ist von zentraler Bedeutung in der Industrie. Unvorteilhafte Prozesssituationen und für die Maschine $u$. $U$. kritische Betriebszustände können durch kontinuierliche Beobachtung von Sensorsignalen sowie daraus resultierenden Handlungsempfehlungen systematisch vermieden und hohe Qualitätsstandards erreicht werden [1]. In der Wertschöpfungskette ist dies speziell auch für Markieranlagen zur individuellen Produktkennzeichnung von Interesse, deren Signatur einerseits die betriebsinterne Rückverfolgbarkeit des Produkts sicherstellt sowie andererseits die eindeutige Identifikation der Warenlieferung und den hochwertigen Markenauftritt beim Endkunden garantiert.

Digitalbilder der Signierung, die z. T. direkt von modernen Markieranlagen archiviert werden, dienen als Basis zur Bewertung des Schriftbildes sowie als Indikator für Fehlerfälle der Anlage. Bei der abgelichteten Markierung (s. Abb. 1) handelt es sich um einen alphanumerischen Punkt-Matrix Aufdruck in weißer
\end{abstract}

Farbe auf einem Grobblech, wobei der Signiertext jedes Bleches einzigartig ist und sich ein Signierblock aus maximal vier Signierzeilen zusammensetzt. Hierbei können negative Einflüsse auf das Signierergebnis, wie beispielsweise Farbmangel oder -überschuss, sowohl lokal stark begrenzt als auch über größere Bildbereiche auftreten, was eine Analyse des Signierergebnisses auf verschiedenen Bildebenen erfordert.

\section{Methodik}

Zur Detektion unerwünschter Effekte werden aus den Bildern daher zuerst sukzessive kleinere Bildausschnitte isoliert und daraus $u$. a. statistische und formbeschreibende Bildmerkmale extrahiert. Da jede Signatur individuell ist, wird eine für das maschinelle Lernen nutzbare Datenbasis aus den wiederkehrenden Schriftzeichen aggregiert. Die Einzelzeichen werden dafür über ein mehrstufiges Segmentierverfahren extrahiert. Durch Kenntnis der Textvorgabe und der herstellerspezifischen Schriftart können darüber hinaus Bildmerkmale auf Signierzeichen- oder -zeilenebene synthetisch generiert werden. Durch die modellbasierte Vorhersage und Differenzbildung von Ortsreihen können Bildattribute, wie z. B. Farb- 
homogenität oder Positionstreue der Signierpunkte, objektiv bemessen werden und die Datenbasis gezielt mit für das maschinelle Lernen relevanten Mustern ergänzt werden.

Die weitere Verarbeitung der Bildsignale erfolgt in den für das maschinelle Lernen typischen Schritten der Merkmalsextraktion, Merkmalsselektion, Modellbildung (Training) und Modellvalidierung (Test). Großes Potenzial liegt hierbei in der signaturunabhängigen Feststellung und Lokalisierung sowohl diskreter Farbeffekte als auch Farbgradienten des Schriftbildes, die von Oberflächendefekten und Umgebungseinflüssen abzugrenzen sind. Entsprechend des Klassifikationsergebnisses und der Anlagensystematik können automatisch typische Ursachen der auftretenden Bildfehler diagnostiziert und Markierungen, die nicht den Anforderungen entsprechen, detektiert werden.

\section{Markieranlage}

Bei der betrachteten industriellen Signieranlage handelt es sich um einen Portal-Aufbau in der Linie oberhalb der Fördereinrichtung der Produkte, was eine Farbsignierung der Bleche im Produktionsfluss gewährleistet. Die Markiereinheit der Anlage befindet sich auf Schlitten an einer Traverse und ist quer zur Transportrichtung des Bleches verfahrbar. Die insgesamt vier Markierköpfe der Markiereinheit können jeweils einer Signierzeile zugeordnet werden. Die Digitalkamera zur Aufnahme der Fotos ist starr an der Markiereinheit der Anlage befestigt und senkt sich mit der Markiereinheit auf eine feste Höhe über die Oberfläche ab. Während des Blechtransports werden mehrere Einzelbilder im Signierprozess angefertigt, die ausschnittsweise die Signatur zeigen und softwaregestützt zur Abbildung der vollständigen Signatur zusammengesetzt werden. Die verfügbare Bilddatenbasis enthält die Archivaufnahmen der Signaturen.

\section{Blechfarbmarkierung}

Die im Fokus der Untersuchung stehende Signatur dient der farblichen Oberflächenmarkierung von Blechen. Die weiße Signatur erstreckt sich über bis zu drei in Leserichtung aufeinanderfolgende Blöcke mit maximal vier Zeilen, die jeweils bis zu 52 Zeichen umfassen können. Der zur Verfügung stehende Zeichensatz ist alphanumerisch und setzt sich aus Groß- und Kleinbuchstaben des lateinischen Alphabets und Sonderzeichen zusammen. Es handelt sich um eine herstellerspezifische Schriftart, wobei ein einzelnes Zeichen in einer Punktmatrix des Formats $10 \times 6$ (bzw. $10 \times 11$ Halbe) abgebildet wird. Die tatsächliche Zeichenhöhe und -breite ergibt sich aus der Anordnung der Punkte zur Darstellung des
Zeichens. Der Abstand der Punkte in der Ebene ist innerhalb des Zeichens konstant. Das Druckraster sieht eine äquidistante Anordnung der Zeichen für eine Signatur vor, d. h. die Zeichen werden zeilenweise gleichmäßig untereinander ausgerichtet. Die Breite eines einzelnen Blockes resultiert aus dessen längster Signaturzeile. Die Größe der Zwischenräume der Signierblöcke ist konstant. Die Markierung der Bleche dient zur eindeutigen Identifikation, weshalb jede Signatur einzigartig ist und folglich nur einmal in der Datenbank vorkommt.
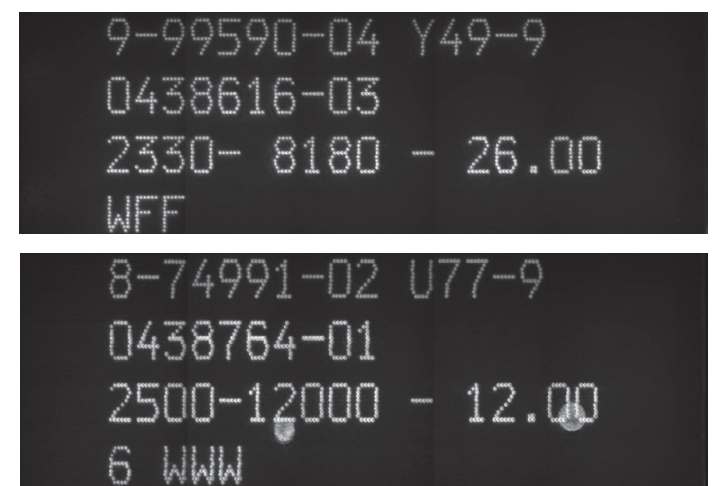

Abb. 1: Beispielhafte Kameraaufnahmen der Blechmarkierung; oben: Signatur ohne Auffälligkeiten, unten: Signatur mit Störeffekten (s. Zeile 3)

\section{Bildverarbeitung}

Laut [3] ist "die industrielle Bildverarbeitung angesichts steigender Anforderungen an Produktqualität und -dokumentation zu einer Schlüsseltechnologie geworden“. Die digitale Bildverarbeitung von Bilddaten stellt typischerweise eine Kette von Bildoperationen dar, die auf den jeweiligen Anwendungsfall spezialisiert ist. Die hier zur Vorbereitung für das maschinelle Lernen ausgewählten Schritte der Bildverarbeitung wurden in der MATLAB Entwicklungsumgebung implementiert und sind nachfolgend kurz genannt. Allgemein werden zur Oberflächeninspektion der Produktmarkierung mehrere Bildvorverarbeitungsschritte zur Erhöhung der Zuverlässigkeit und Stabilität bei der Objektlokalisation und -qualitätsbestimmung durchgeführt, wie sie auch für die Erkennung von Barcodes in [3] beschrieben wird. Zuerst wird eine Bildglättung vorgenommen, welche die Struktur des Bildes weichzeichnet und die Bildverarbeitung in der weiteren Abfolge unempfindlicher gegenüber Bildrauschen macht. Weiterhin kann durch eine Normierung des Bildes in Form einer Histogrammspreizung und -verschiebung eine Kontrastverbesserung erreicht werden. Der hierbei verwendete Normierungsfaktor, ein Maß für die Bildhelligkeit, der potenziell 
relevante Information für die spätere Klassifikation der Bilddaten enthält, wird als Einzelmerkmal gespeichert.

\section{Zeichensegmentierung}

Prinzipiell werden für das maschinelle Lernen Datenbestände benötigt, die für je eine Klasse ähnliche Merkmale aufweisen und eine Mustererkennung der Daten zulassen, sodass eine Abgrenzung zwischen den Klassen vollzogen werden kann. Für die hier betrachtete Anlagenzustandsüberwachung bedeutet dies, dass für die verschiedenen Prozesssituationen ausreichend Bildaufnahmen vorliegen müssen. Da allerdings jede der Markierungen individuell ist und inhaltlich, d. h. auch optisch, stark variiert, ist eine Verallgemeinerung der Bilddaten ungeeignet. Demzufolge ist auf Basis der Signaturaufnahmen vorerst keine Trendanalyse sinnvoll. Um dennoch auf einen hinreichenden Probenumfang für das Training beim maschinellen Lernen zurückgreifen zu können, werden die Einzelzeichen der Signaturen extrahiert und verglichen. Durch Betrachtung der Bildausschnitte der Signierzeichen bleibt der Eindruck der Schriftbildqualität erhalten und die zur Verfügung stehende Datenbasis wird vervielfältigt und abstrahiert.

\section{Segmentierung}

Für die Segmentierung der Zeichen wird die Information zur Lage des Objekts innerhalb der Bildebene gesucht. $\mathrm{Da}$ die gedruckte Markierung grundsätzlich einer festen Gestaltungsvorgabe unterliegt und die Bildauflösung konstant ist, kann bereits ein grober Umriss des Interessensbereichs, der die Signatur beinhaltet, vorgenommen werden. Die anschließende Bildvorverarbeitung dient der Vereinfachung der Trennbarkeit von Vordergrund (Signatur) und Hintergrund (Blechoberfläche). Diesbezüglich werden u. a. morphologische Bildoperationen zur Verstärkung und Zusammenführung der Konturen der Signierpunkte vorgenommen, um einzelne Zeichen als zusammenhängend zu identifizieren. Gemäß der horizontalen Ausrichtung der Signatur kann im nächsten Schritt durch zeilenweise Integration der Pixelwerte und statische Schwellwertsetzung die Anzahl und Intervalle der Zeilenindizes ermittelt werden (s. Abb. 2) Die Länge eines Intervalls entspricht dabei der jeweiligen Höhe der Signaturzeile bzw. der dazugehörigen Einzelzeichen. Es wird weiterhin kontrolliert, ob das Pixelmaß der Zeilenhöhen und der vertikalen Zeilenabständen innerhalb des plausiblen Toleranzbereichs liegt, da die detektierte Höhe einer Signaturzeile typischerweise leicht schwanken kann.
Um zur besseren Vergleichbarkeit der Zeichen nun Bildsegmente gleicher Größe aus dem Gesamtbild zu isolieren, wird nur der Zeilenindex der positiven Flanke, d. h. des theoretisch ersten Bildpixels, das die Signierzeile abbildet, übernommen und eine feste Intervallänge angenommen. Angesicht der linksbündigen Ausrichtung und dem festen MatrixFormat der Signierzeichen kann ebenso in Spaltenrichtung zur Bestimmung der horizontalen Zeichenlage und -breite verfahren werden.
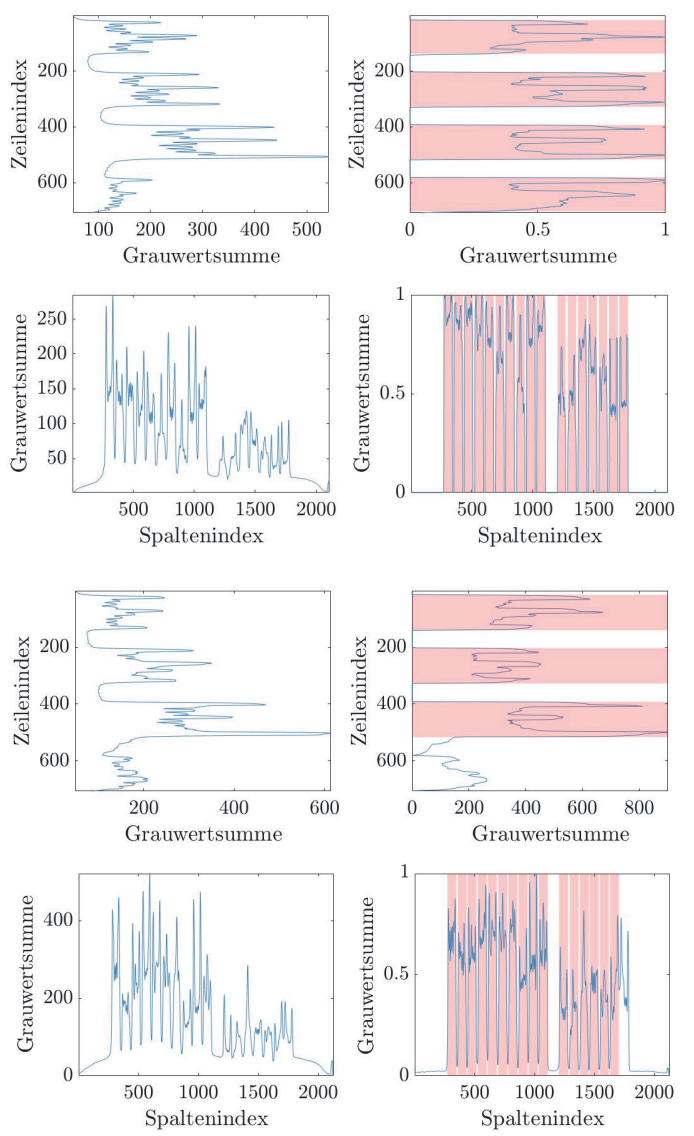

Abb. 2: Zeilen- und Spaltensignale (links) sowie deren Anpassung (rechts) zur Segmentierung der Signierblöcke in Einzelzeichen;

oben: erstes Bild aus Abb. 1, unten: zweites Bild aus Abb. 1

Jedes der dadurch gewonnenen Wertepaare mit den Vektoren der Zeilen- und Spaltenindizes impliziert eine potenzielle Position eines Bildabschnitts mit einem Signierzeichen. Da jedoch die Spalteninformation komprimiert über alle Zeilen erworben wird, d. h. eventuell nur von einem einzigen Zeichen in dieser Signierspalte stammen kann, ist nicht gegeben, dass alle potenziellen Bildsegmente mit Zeichen besetzt sind. Um dies zu überprüfen, wird für die weitere Verwendung die jeweilige Zeichenposition mit dem vorliegenden Solltext verglichen und für den Fall einer Leerstelle ver- 


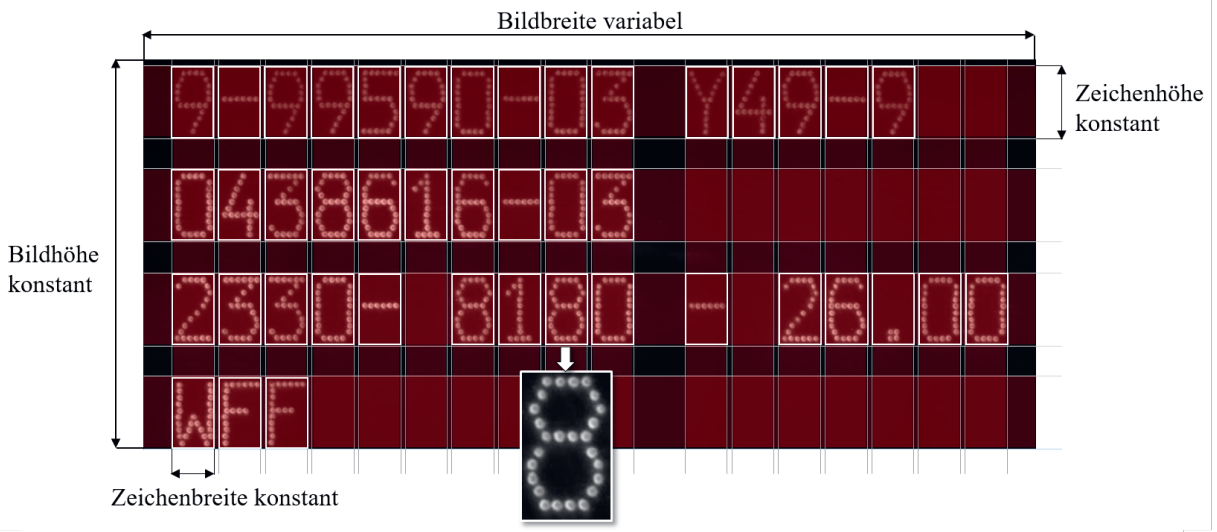

Abb. 3: Grafische Darstellung der Segmentierung von Einzelzeichen eines Signierblockes im Interessensbereich mit Kennzeichnung der Geometrieverhältnisse

worfen. Mittels der verbleibenden Lagebeschreibungen kann der relevante Bildabschnitt erschlossen und separat als Bilddatei zu einer Datenbasis hinzugefügt werden (s. Abb. 3).

Neben der Bildverarbeitung zur Objektlokalisation und Bereitstellung der Bildsegmente für das maschinelle Lernen dient der Algorithmus zur Extraktion der Einzelzeichen gleichzeitig auch als erster Indikator für die Qualität des Schriftbildes der Signatur. Da die Segmentiervorschrift an den Aufbau einer Standard-Signatur angepasst ist, verläuft die Routine nur bei erwartungsgemäßen Signaturen erfolgreich. Falls das Signaturlayout nicht dem hinterlegten Profil entspricht, d. h. Signierzeilen mit der Programmlogik nicht unmittelbar erkannt werden oder Abstände nicht der Plausibilitätsprüfung genügen, liegt offensichtlich eine Anomalie vor, die eine Qualitätseinbuße bedeutet (vgl. Abb. 2 unten). Dieses Abbruchkriterium wird als Indiz festgehalten und in Abhängigkeit dessen die möglichen Ursachen für die Abweichung bestimmt.

\section{Erstellung einer Referenzdatenbasis}

In dieser Arbeit wird ein neuartiger Ansatz zur künstlichen Generierung von Trainingsdaten verfolgt. Es wird überprüft, inwiefern sich synthetische Bilddaten, welche verschiedene Prozesslagen der Maschine möglichst optimal nachbilden, zur Kategorisierung der Archivaufnahmen eignen. Im Rahmen der Bildverarbeitung wird zu diesem Zweck ein Modell entwickelt, das entsprechend dem Muster der vorliegenden Signaturbilder möglichst realitätsnah synthetische Signaturbilder generiert. In Anlehnung an die typografische Gestaltung, das Farbschema und den geometrischen Aufbau der Blechmarkierung kann sukzessive das Einzelzeichen und weiterführend die Signaturzeile bzw. Gesamtsignatur künstlich erzeugt und zusammengefügt werden.

\section{Modellbilder}

Methodisch wird zuerst eine zweidimensionale Matrix erstellt, deren Größe sich an der Auflösung der Zeichensegmente der Archivbilder orientiert und deren Elemente den Grauwert für den Hintergrundfarbton der Signatur enthalten. Anschließend wird im Abgleich mit den vorliegenden Fotobeispielen das Punktraster ermittelt, welches die möglichen Positionen der Kreismittelpunkte für die Zeichen in Punkt-Matrix-Schriftart wiedergibt. Die benötigte Pixelanzahl für den Durchmesser wird aus den Fotobeispielen bestimmt. Die Farbverteilung der einzelnen Signierpunkte wird durch die zweidimensionale Projektion einer Halbkugel abgeleitet, deren Höhenausprägung entlang der rotationssymmetrischen Achse den Helligkeitswert angibt. Gemäß der bekannten Anzahl und Anordnung der Signierpunkte pro Zeichen werden diese iterativ im Bildraster ausgerichtet und an die passende Stelle projiziert, wo die Grauwerte der Bildmaske des Signierpunktes zu den relevanten Matrixeinträgen der Hintergrundmaske addiert werden. Das auf diese Weise modellierte Bild wird abschließend mit einem Gauß-Filter weichgezeichnet, sodass harte Konturen geglättet werden und das Bild der realen Fotoaufnahme ähnlicher erscheint. Beispielbilder sind im Abschnitt Zusammenstellung der Klassen aufgeführt.

\section{Manipulation der Modellbilder}

Die Standardausführung der Modellbilder kann nun in der Folge so manipuliert werden, dass die synthetischen Daten Signierfehler imitieren, die verschiedene Degradationsstufen der Schriftbildqualität repräsentieren. Die Bilddefekte sollen wiederum den entsprechenden kritischen Prozesssituationen zugeordnet werden können, wodurch von der Signaturqualität auf den Anlagenzustand geschlossen 
werden kann. Das heißt, dass durch gezielte Variation der Modellbilder nach Signaturvorbild mangelbehafteter Exemplare, die aus seiner bestimmten Anlagenstörung resultieren, eine entsprechende Referenzdatenbasis für die Fehlerklassen generiert werden kann. Zu den skalierbaren Modellparametern der Signierpunkte zählen:

$$
\begin{aligned}
& \text { - Helligkeit } \\
& - \text { Schattierung } \\
& - \text { Größe } \\
& - \text { Exzentrizität } \\
& - \text { Homogenität } \\
& - \text { Kontrast } \\
& -\quad \text { Positionstreue. }
\end{aligned}
$$

Die Helligkeit, welche der Intensität der Signierfarbe bzw. deren Reflexion gleich kommt, kann durch Vorgabe des Maximalwertes für die Bildmaske des Signierpunktes angepasst werden. Das Strukturelement für die Nachbildung des Signierpunktes, hier als Schattierung bezeichnet, kann neben der genannten Halbkugel auch einer anderen Funktion, z. B. einer Gauß-Verteilung, folgen. Die Größe eines projizierten Signierpunktes wird durch Angabe des gewünschten Radius in Pixel eingestellt. Das Format der Bildmaske muss dabei aber nicht quadratisch sein, sondern kann auch in Zeilenrichtung verzerrt sein, was einer Erhöhung der Exzentrizität, d. h. der Abbildung einer Ellipse, gleichkommt. Diese Deformation der Kreisgeometrie wird z. B. durch morphologische Dilatation erreicht. Die Homogenität eines modellierten Signierpunktes, die zum einen durch die Farbhöhen und zum anderen durch die lokale Betrachtung der Pixelumgebung bestimmt wird, kann durch die Überlagerung mit Rauschsignalen verändert werden. Der Kontrast der Signierpunkte, d. h. die Stärke des Gradienten am Rand des Objektes, welche maßgeblich für den Schärfeeindruck eines Bildes ist, kann durch die Operation des Weichzeichnens gemildert werden. Auch das Angleichen der Hintergrundhelligkeit verändert dieses Bildattribut. Zur Beeinflussung der Positionstreue der Punkte im ermittelten Raster der PunktMatrix-Schriftart kann eine Anpassung der Zeilen- und Spaltenindizes erreicht werden. Die Änderung des Kreisursprungs resultiert in einer Abweichung der Lage des modellierten Signierpunkts in der Zeichenebene.

Die beschriebenen Bildmanipulationen können je nach gewünschter Art des Bilddefekts einzeln oder in beliebiger Kombination durchgeführt werden. Die Ausprägung der Defekte kann innerhalb eines Toleranzbereichs zufällig erfolgen oder auch abgestimmt auf einen speziellen Fehlerfall.

\section{Bildmerkmale}

„Das Auge erfaßt Bilder eher qualitativ, während die quantitative Extraktion von Bildmerkmalen eine quantitative Analyse der Filtereigenschaften erfordert." heißt es in [4], was die Subjektivität des Qualitätseindrucks eines Bildes widerspiegelt und die Sorgfalt bei der Auswahl von Bildoperationen zur Merkmalsextraktion betont. Ziel bei der Dimensionsreduktion der Bilddaten ist es, charakteristische Bildeigenschaften möglichst kompakt darzustellen und gleichzeitig wenig der für die Beurteilung der Qualität relevanten Information über Filteranwendungen zu verlieren. Da die Bestimmung und Interpretation der aussage-

\begin{tabular}{|c|c|}
\hline Merkmalstyp & Beispiele \\
\hline Spaltenmerkmal & \multirow{2}{*}{$\begin{array}{l}\text { Summen der Grau- } \\
\text { wertpixel/ Binärpixel/ } \\
\text { Kantenpixel, Mittelwert, } \\
\text { Varianz }\end{array}$} \\
\hline Zeilenmerkmal & \\
\hline $\begin{array}{l}\text { Histogramm- } \\
\text { merkmal }\end{array}$ & $\begin{array}{l}\text { Bild vor/ nach } \\
\text { Kontrastanpassung, } \\
\text { Differenzbild aus } \\
\text { Kontrastanpassung }\end{array}$ \\
\hline Objektmerkmal & $\begin{array}{l}\text { Mittelpunkt/ Form/ } \\
\text { Fläche des Objekts }\end{array}$ \\
\hline Kreismerkmal & $\begin{array}{l}\text { Radius, Mittelpunkt, } \\
\text { Sensitivität der } \\
\text { Kreisdetektion }\end{array}$ \\
\hline Sekundärmerkmal & $\begin{array}{l}\text { Faktor der Kontrast- } \\
\text { anpassung, Anzahl der } \\
\text { Objekte/ Kreise }\end{array}$ \\
\hline
\end{tabular}
kräftigen Bildattribute jedoch Gegenstand der Forschungsarbeit ist, gilt es im ersten Iterationszyklus, möglichst viele Bildmerkmale zu bestimmen, die weiterführend analysiert und selektiert werden können.

\section{Tab. 1: Einteilung der Bildmerkmale}

Bei der durchgeführten Merkmalsextraktion werden entsprechend der Herkunft der Information bislang sechs verschiedene Merkmalstypen unterschieden, wie in Tabelle 1 gelistet. Spalten- bzw. Zeilenmerkmale beziehen sich auf die Verarbeitung der Ortsreihen entlang der jeweiligen Richtung. Diese werden $u$. a. durch statistische, formbeschreibende und spektrale Signalanalyse gewonnen und sind für beide Dimensionen äquivalent durchführbar. Im Zuge dessen können sowohl absolute als auch relative, d. h. normierte, Merkmale für die Auswertung herangezogen werden. Diese können wiederkehrend nach verschiedenen lokalen und globalen Bildoperationen für die aktuelle Ausführung des Bildes bestimmt werden, was das Angebot an potentiell wichtigen Merkmalen steigert. Gleiches gilt auch für 
die Einbindung der Histogramme als densitometrische Merkmale für verschiedene Versionen des Bildes, die Aufschluss über Farbhäufungen innerhalb eines Bildes bzw. eines Teilsegments geben. Diese können chromatisch sortiert sowie kumuliert dargestellt werden. Durch beliebige Skalierung der Klassenbreite kann die Anzahl an Einträgen des Merkmalsvektors variiert werden.

Weiterhin werden Objekt- und Kreismerkmale bestimmt, die sich auf eine definierte Region beziehen. Als Objekt wird in diesem Kontext eine zusammenhängende Pixelnachbarschaft verstanden, die nach einer mehrstufigen Vorverarbeitung ein Schwellwertkriterium erfüllen. Im vorliegenden Fall der Signaturzeichen handelt es sich dabei i. d. R. um unidirektional verknüpfte Signierpunkte. Die Gestalt und Ausrichtung eines Objektes sowie der Objekte zueinander, ebenso wie u.a. die ursprünglichen Werte der assoziierten Bildpixel, bildet eine Merkmalskategorie zur Erfassung der Topologie und Struktur des Bildes ab. Weitere Merkmalstypen, die zur Veranschaulichung von Lage, Geometrie und Kolorit der Signierpunkte dienen, sind die Kreismerkmale. Da es sich bei der vorliegenden Schriftart der gedruckten Zeichen um eine Punkt-Matrix-Schrift handelt, ist demzufolge eine Isolation der runden Zeichenbausteine zur Beurteilung ihrer Ähnlichkeit naheliegend. Da in Bezug auf den Signierprozess die Punktzeilen jeweils einer eigenen Markierdüse zugeordnet werden können, d. h. im Falle von Unstimmigkeiten die wahrscheinliche Ursache eingegrenzt werden kann, ist diese Eigenschaft im Hinblick auf den Anlagenzustand von besonderem Interesse. Die Routine zur Identifikation der runden bzw. kreisähnlichen Bilderhöhungen ist der proprietären Image Processing Toolbox entnommen. Zur effizienten und robusten Detektion der Signierpunkte sind Vorverarbeitungsschritte, welche den Algorithmus der Kreiserkennung begünstigen, erforderlich. In einer Programmschleife werden zu diesem Zweck Kontrastund Konturanpassungen vorgenommen, bis die gegebene Maximalzahl von Signierpunkten erreicht ist. Sind die idealen Umrisse der Signierpunkte ermittelt, kann eine Auswertung der einbeschriebenen Pixel im Originalbild (s. Abb. 4) und weiteren Bildausführungen erfolgen. Dabei impliziert die Abweichung der Radien und Koordinaten z. B. ein Maß für die Prozessstreuung respektive die Positionstreue der Signierpunkte.

Weiterhin werden im Zuge der Bildverarbeitung stellenweise Einzelwerte gesammelt, die in der Gruppe der Sekundärmerkmale zusammengefasst werden, und sich als zusätzliche Deskriptoren für die Bilder eignen. Zu diesen
Metadaten zählen beispielsweise Angaben über die Anzahl der von Null verschiedenen Einträge eines Merkmalsvektors, das Integral einer Bildregion oder Filterparameter.

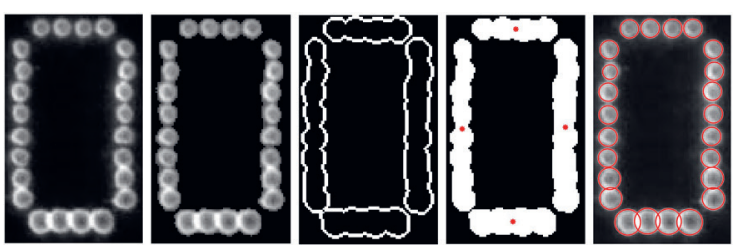

Abb. 4: Bildversionen der Bildverarbeitungskette: v.l.n.r. (1) Originalbild, (2) Freistellung des Vordergrundes, (3) Kantenbild, (4) Binärbild mit Kennzeichnung der Objektmittelpunkte, (5) Originalbild mit Projektion der detektierten Kreise

Die aufgeführten Bildmerkmale, als kompakte und charakteristische Repräsentation der Aufnahmen der Signaturzeichen, werden anschließend mit Methoden des maschinellen Lernens auf Trennbarkeit untersucht.

\section{Maschinelles Lernen mit Bilddaten}

Die Überführung der Bildinformation in unterscheidbare Merkmale dient zum einen der Reduktion des hochdimensionalen Merkmalsraums von Fotoaufnahmen und zum anderen der Projektion von Gemeinsamkeiten zur Aggregation für das maschinelle Lernen. Die aus dem Produktionsprozess verfügbaren Bilddaten der Signatur werden durch Kombination der genannten Bildverarbeitungsschritte dem maschinellen Lernen zugänglich gemacht.

\section{Zusammenstellung der Klassen}

Zunächst wird durch manuelle Vorauswahl von Bildexemplaren, die subjektiv und erfahrungsgemäß einer guten bzw. einer schlechten Signierbildqualität entsprechen, eine Datenbasis zur Erkennung von einwandfreien und kritischen Bildern zusammengestellt. Da die Information zur Wertigkeit des Markierergebnisses zwar zeichenunabhängig ist, sich die Bildinformation der Zeichen aber aufgrund der Zeichengestalt stark unterscheidet und somit keine Ähnlichkeit innerhalb der Qualitätsklassen gegeben ist, werden für das maschinelle Lernen die Signaturzeichen getrennt betrachtet. Dadurch sollen die fehlerhafte Interpretation und das Training auf systematische Zeichenunterschiede unterbunden werden. Da jedoch für das maschinelle Lernen eine möglichst große Datenbasis ideal, die manuelle Selektion aber sehr zeitaufwändig ist, soll nach dem Muster der Archivdaten eine modellbasierte Referenzdatenbasis generiert werden, die eine automatische Klassifizierung 
der Zeichensegmente ermöglicht. Dazu werden zunächst Modellbilder anhand der Parameter, wie im Abschnitt Manipulation der Modellbilder beschrieben, zur Imitation der Kategorie der guten Beispiele (,i. O.") und der schlechten Beispiele („n. i. O.“) angepasst. Die Abbildungen 5 und 6 zeigen eine Teilmenge der jeweiligen Kategorien.

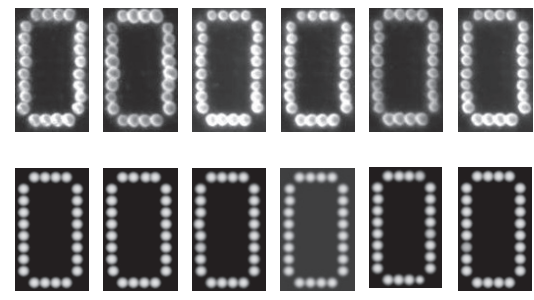

Abb. 5: Bildbeispiele der Klasse „i. O.”; oben: Originalbilder aus dem Archiv, unten: Modellbilder aus der synthetischen Datenbasis
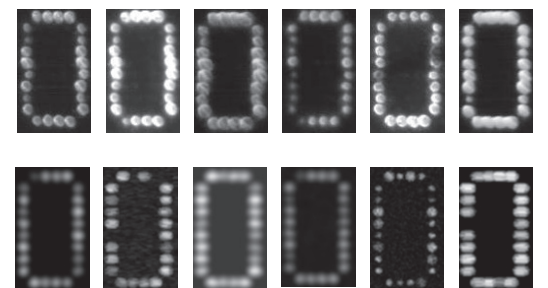

Abb. 6: Bildbeispiele der Klasse „n. i. O.”; oben: Originalbilder aus dem Archiv, unten: Modellbilder aus der synthetischen Datenbasis

\section{Multivariate Datenanalyse}

Wie u.a. in [5] erwähnt, ist für das Konzept des maschinellen Lernens bekannt, „dass die Wahl des Verfahrens immer anhand empirischer Untersuchungen und den technischen Anforderungen [...] der Aufgabenstellung getroffen werden muss". Vor diesem Hintergrund wurden mithilfe der in [2] beschriebenen Methoden Vergleichsstudien zu den für die Klassentrennung bedeutsamen Merkmalen und deren Einfluss auf das Klassifikationsergebnis durchgeführt. Für das vorliegende Szenario erfolgt das maschinelle Lernen überwacht, d. h. die Klassenzugehörigkeit der Bilder ist gegeben. Wie im Abschnitt Zusammenstellung der Klassen erläutert, gibt es testweise zwei heterogene Klassen „i. O.“ und „n. i. O.“, die aus derselben Anzahl (je 100 Exemplare) an Original- und klassenspezifischen Modellbildern bestehen.

Das für das vorliegende Szenario beste Ergebnis zur Trennung der Klassen wurde mit dem zweistufigen Konzept der Merkmalsselektion mittels Pearson Korrelation und Recursive Feature Elimination Support Vector
Machines (RFESVM) erreicht. Dabei wird aus Effizienzgründen zuerst eine Vorauswahl von 500 Merkmalen getroffen [1] und diese anschließend hinsichtlich ihrer Eignung zur Trennung der Klassen schrittweise verworfen. Dabei wird die Redundanz von Merkmalen berücksichtigt. Details des Algorithmus sind [6] zu entnehmen.

Die minimale Fehlerrate bei 10-facher Kreuzvalidierung und Klassifikation mit dem kNächste-Nachbarn-Verfahren $(\mathrm{k}=1)$ beträgt $5,75 \%$ bei Betrachtung der zwölf besten Merkmale. Der Verlauf des Klassifikationsfehlers als Funktion der Merkmalsanzahl ist in Abb. 7 gezeigt. Zu den ausgewählten Merkmalen zählen Bildattribute aus den Kategorien Zeilen-, Spalten-, Histogramm-, Objekt- und Kreismerkmal, siehe Tabelle 1.

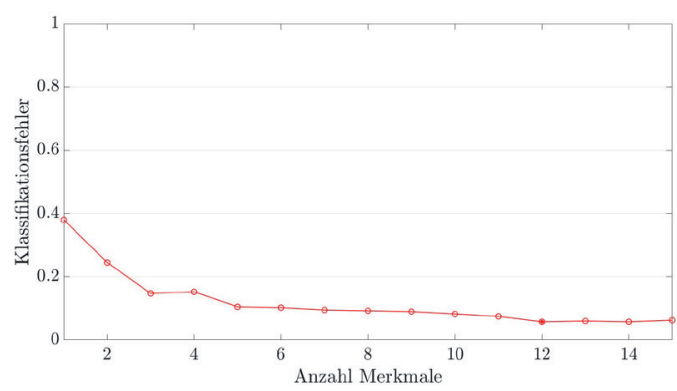

Abb. 7: Darstellung des Klassifikationsfehlers bei Klassifikation mit $1 \mathrm{NN}$-Methode

Das Projektionsergebnis einer linearen Diskriminanzanalyse (LDA) basierend auf den zwölf besten Merkmalen ist in Abb. 8 dargestellt. Das Ergebnis zeigt, dass die beiden Gruppen „i. O.“ und „n. i. O.“ sowohl für Modell- als auch Originalbilder im Mittel gut getrennt werden. Auffällig ist, dass die „i. O.“ Modellbilder eine deutlich geringere Variation des Diskriminanzwertes zeigen als die drei anderen Untergruppen.

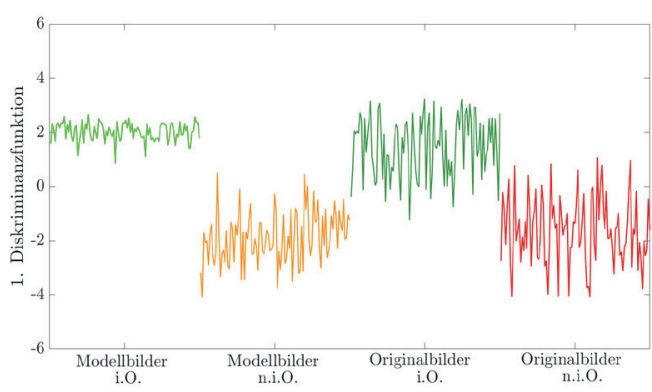

Abb. 8: Darstellung der Diskriminanzfunktion für die besten 12 Merkmale nach Vorselektion mittels Pearson Korrelation und Merkmalsselektion mittels RFESVM für die Bildgruppen der Klassen „i. O.” und „n. i. O.” 


\section{Diskussion und Ausblick}

In Abbildung 8 ist zu erkennen, dass die Variablen der Originaldaten der Klassen z. T. treffend durch die Modellbilder nachempfunden werden. Jedoch wird deutlich, dass keine eindeutige Trennbarkeit erreicht wird und die Variation der Modellbilder insbesondere für die Klasse „i. O.“ noch nicht hinreichend dem Ideal der Originalbilder entspricht. Daher sollen die Modellbilder der Referenzdatenbasis hinsichtlich Authentizität und Repräsentationsvermögen weiter optimiert und zusätzlich die Extraktion der Bildmerkmale erweitert werden, um den Klassifikationsfehler zu minimieren.

Darüber hinaus handelt es sich bei der Modellierung der Aufgabenstellung als 2-KlassenProblem bis dato um eine grobe, unzulängliche Formulierung der beobachteten Qualitätsabstufungen, welche mit den verwendeten Verfahren der Merkmalsselektion nur bedingt erfasst werden kann. Dies soll durch eine feinere Unterteilung der kritischen Bilderklasse „n. i. O." verbessert werden. Eine Klassenaufspaltung und Ableitung von Handlungsempfehlungen gemäß der Systemursache der Anomalien erfordert dementsprechend eine Gruppierung der Bilddefekte nach Störquellen.

\section{Fazit}

Die bisherige Forschungsarbeit zeigt ein Konzept zur Beurteilung der Schriftbildqualität von Blechmarkierungen in Punkt-Matrix-Schrift und erläutert die Modellbildung von Referenzdaten zur Erweiterung der verfügbaren Datenbasis für das Training im maschinellen Lernen. Nach einer mehrstufigen Merkmalsextraktion und -selektion wird mittels Nächste-NachbarnKlassifikation ein minimaler Klassifikationsfehler von 5,75\% erzielt. Aufbauend auf diesen Ergebnissen laufen aktuell Arbeiten zur anlagenzustandsadäquaten Erweiterung der Klassen und zur realitätsnahen Optimierung der Modellbilder.

\section{Literaturnachweis}

[1] VDMA, Future Manufacturing: Magazin für intelligente Produktion, VDMA Verlag (2017/03)

[2] T. Schneider, N. Helwig, A. Schütze, Industrial condition monitoring with smart sensors using automated feature extraction and selection, IOP Meas. Sci. Technol. (2018) 29 094002, doi: 10.1088/1361-6501/aad1d4

[3] C. Demant, B. Streicher-Abel, A. Springhoff, Industrielle Bildverarbeitung - Wie optische Qualitätskontrolle wirklich funktioniert, Springer Berlin Heidelberg (2011), doi: 10.1007/978-3-642-13097-7
[4] B. Jähne, Digitale Bildverarbeitung und Bildgewinnung, Springer Vieweg (2012), doi: 0.1007/978-3-642-04952-1

[5] H. Süße, E. Rodner, Bildverarbeitung und Objekterkennung - Computer Vision in Industrie und Medizin, Springer Vieweg (2014), doi: 10.1007/978-3-8348-2606-0

[6] I. Guyon, M. Nikravesh, S. Gunn, L. A. Zadeh, Feature Extraction Foundations and Applications, Springer Berlin Heidelberg (2006), doi: 10.1007/978-3-540-35488-8 